\title{
Transfer from Pediatric to Adult Care Is Hardly Child's Play
}

\author{
Amanda Steiman $^{1}$ (D) and Zahi Touma ${ }^{\text {(D) }}$
}
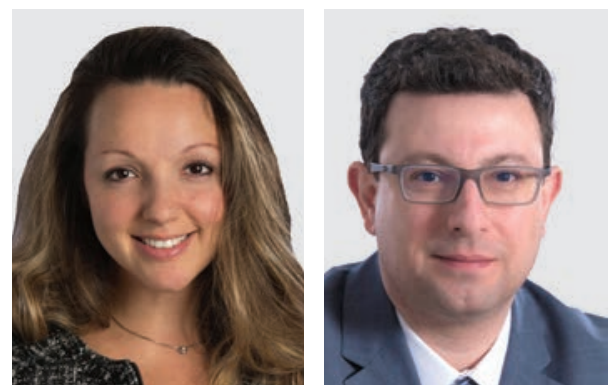

In this issue of The Journal, Chang and colleagues describe patterns of rheumatologic/nephrologic care and changes in healthcare use and medication adherence among patients with childhood-onset systemic lupus erythematosus (SLE) during their transfer from pediatric to adult care ${ }^{1}$. The most salient of their findings was a $26 \%$ rate of loss to follow-up, defined as more than 12 months between last pediatric visit and first adult visit, or end of enrollment. Consequently, and not surprisingly, ambulatory visits declined (although interestingly, there was no signal for increased acute care use). Medication adherence was poor across groups, regardless of whether the transfer was deemed a "success."

Taken together, Chang, et als findings reinforce the overarching themes of the literature on pediatric transition to adult care, not to mention the anecdotal - but remarkably consistently held - impressions of physicians who practice in this space: that the period of transition from pediatric to adult care is littered with landmines, quilted with quagmires. Some find their way to adult care and succeed; many others, however, fail dramatically and with dire consequences. Other groups have reported that only $50 \%$ of pediatric rheumatology patients transition successfully to adult care ${ }^{2,3}$. Surely, we could forge a less perilous path.

And while patients with childhood-onset SLE, like those in the Chang study, reflect a particularly vulnerable group, with complex and potentially fatal multisystemic disease, the challenges associated with transfer from pediatric to adult care transcend diagnosis or specialty. Themes are consistent among

${ }^{1}$ A. Steiman, $M D, M S c, F R C P C$, University of Toronto, Department of Medicine, Division of Rheumatology, Sinai Health System/University Health Network; ${ }^{2} Z$. Touma, MD, PhD, FACP, FACR, University of Toronto, Department of Internal Medicine, Division of Rheumatology, Centre for Prognosis Studies in the Rheumatic Diseases, Toronto Western Hospital, Toronto, Ontario, Canada.

Address correspondence to Dr. A. Steiman, Sinai Health System, Medicine, 60 Murray St., Suite 2-223, Toronto, ON MST 3L9, Canada.

Email:amanda.steiman@sinaihealthsystem.ca. chronic diseases of onset in childhood that persist into adulthood regardless of whether the young patient has SLE, juvenile idiopathic arthritis (JIA), diabetes, or sickle cell disease ${ }^{4,5,6}$.

The unique challenges associated with care of these patients are driven by their context. These patients present carrying the physical, psychosocial, and psychic baggage of a chronic disease (whose weight their parents have borne, at least partially) and are now shouldering its bulky burden through the formative experiences that define adolescence. This is a time of differentiation and distancing from parents (sometimes rapidly), progressive independence, moving away from home to attend college or university, experimentation with evolving sexuality, and exposure to drugs and alcohol. Indeed, these formative experiences shape the adults they will become.

For these adolescents with chronic disease, the decision to live away from home must include consideration of the prospect of disease flare and admission to an unfamiliar hospital, far from family. Their decision to live in university residence may affect their ability to manage sleep hygiene, so critical to disease control in many. In others, the intersection between disease process and increased risk associated with oral contraceptives may introduce complex and catastrophic consequences, from unplanned to complicated pregnancies. There is a dizzying array of scenarios wherein disease can affect the experiences, and vice versa.

So how can we collectively catalyze the timely and seamless transition of these young patients into adult care?

1. Pediatric patient preparation.

Patients - nay, humans - fear the unknown. And adult care can reflect the Great Unknown for young adults with SLE and other chronic diseases: patients are now expected to take progressive ownership over illness and its management that, in many cases, was largely managed by parents/caregivers, and to evolve into this role in the uncharted context of adult care. Maddux and colleagues surveyed 370 pediatric patients with inflammatory bowel disease about their perspectives on transition, and found that, among respondents, the most important reasons for unsuccessful transfer, accounting for over half of the responses, were

\section{See Pediatric SLE transition outcomes, page 105}


comfort with their pediatric doctor and worry about starting over with a new physician ${ }^{7}$. Among this cohort, discussion of the differences between pediatric and adult care was ranked second in importance only to discussion surrounding independent management of their illness and its treatment.

Qualitative interviewing of patients with JIA transitioning to adult care extracted familiarization with the adult clinic as an important factor in allowing the focus to be placed on managing their disease, rather than devoting excess energy to a new setting8. Indeed, the simple theme of familiarity with adult care emerges time and time again in transition literature, and could reflect an important opportunity to improve successful coordination of transition, and in doing so, improve outcomes in this vulnerable population $^{9,10}$.

With this in mind, perhaps even simple efforts to assuage the patient's fear and anxiety may be of disproportionately high value in promoting successful transition.

2. Provider preparation.

It was nearly 2 decades ago that the American Academy of Pediatrics (AAP) published a consensus statement on healthcare transitions for young adults with special healthcare needs ${ }^{11}$. Its goal was to ensure that, by 2010, "all physicians who provide primary or subspecialty care to young people with special healthcare needs (1) understand the rationale for transition from child-oriented to adult-oriented health care; (2) have the knowledge and skills to facilitate that process; and (3) know if, how, and when transfer of care is indicated." This has driven campaigns like "Got Transition," which serve as resources for physicians, patients, and their parents/caregivers. In spite of these efforts, however, it seems transition from pediatric to adult care is fraught with challenges and considerable risk.

As adult rheumatologists, our assumption was that pediatric rheumatologists' knowledge of and comfort with the transition process were robust. We were thus surprised by the findings of Chira and colleagues, who in 2014 interviewed members of the Childhood Arthritis and Rheumatology Research Alliance, composed mainly of pediatric rheumatologists (for whom transition of patients is an inevitability), and found that fewer than $10 \%$ were very familiar with the AAP transition guidelines, and only $8 \%$ had a formalized, written transition policy ${ }^{12}$.

It is reasonable to assume, then, that adult rheumatologists, whose patients can range in age from 18 years to 118 years, may feel ill-equipped to navigate the challenges of transitioning young adults from pediatric care. Indeed, adult rheumatologists do not typically receive formal training to prepare for the provision of the unique and stage-appropriate care for patients with pediatric-onset disease, which for many, will persist through their adult lives. Matsui and colleagues surveyed adult rheumatologists about issues interfering with transition from pediatric to adult care. They found that, while the vast majority $(87 \%)$ cared for patients who had transitioned from pediatric care, nearly half reported hesitation and/or anxiety, largely stemming from feeling ill-equipped to address the unique challenges of this patient population ${ }^{13}$.

In recent years, European League Against Rheumatism/
Paediatric Rheumatology European Society (EULAR/PReS) published standards and recommendations on the provision of transitional care for patients with pediatric-onset rheumatic diseases. Acknowledging that application would present a challenge, they organized these by what was felt to be the bare minimum to support successful transition (i.e., "essential"), and that which reflected optimal care (i.e., "ideal") ${ }^{14}$. This may reflect a fruitful common ground to assist in pediatric and adult collaborations in this important field, and generate new knowledge on the approach to and value of dedicated focus on transfer and transition.

It is critical to identify champions on both the pediatric and adult side - the rheumatologists leading Young Adult Rheumatic Disease (YARD) clinics, or others testing alternative models for the delivery of transition care, as was the case in Chang, et als analysis. These champions will serve an invaluable role in studying and adapting models of care to reflect best practice to meet the needs of our young adult patients and ensuring a timely transition to YARD clinics, appropriate follow-up care, and stringent adherence to medications. That said, there is unequivocal value in all rheumatologists, on both the pediatric and adult sides, having facility with the tenets of transition, to promote (at least) "essential" best practices if the luxury of referral to such a specialized clinical model does not exist.

There are consistent differences in the resources and infrastructure available on the pediatric and adult sides, with the latter almost universally being more austere ${ }^{15}$. Nursing, social work, and allied health support, not to mention resources such as patient information sessions and development of IT platforms to better support young adult patients, are perceived as advantages in the provision of care for transitioning young adults, but they are resource- and cost-intensive. The ability to better lobby for support for the implementation of any of these professionals and/or services into transition programs would be bolstered by data on their roles in supporting best practices, as outlined in the EULAR/PReS standards and recommendations.

It seems the old pediatric adage that "children are not just small adults" remains of value in our reflection on the provision of care to young adults. There is no better time than the present to work together to improve these patients' futures.

\section{REFERENCES}

1. Chang JC, Knight AM, Lawson EF. Patterns of healthcare use and medication adherence among youth with systemic lupus erythematosus during transfer from pediatric to adult care. J Rheumatol 2021;48:105-13.

2. Ramos AL, Hoffmann F, Albrecht K, Klotsche J, Zink A, Minden $\mathrm{K}$. Transition to adult rheumatology care is necessary to maintain DMARD therapy in young people with juvenile idiopathic arthritis. Semin Arthritis Rheum 2017;47:269-75.

3. Hazel E, Zhang X, Duffy CM, Campillo S. High rates of unsuccessful transfer to adult care among young adults with juvenile idiopathic arthritis. Pediatr Rheumatol Online J 2010;8:2.

4. Robertson L. When should young people with chronic rheumatic disease move from paediatric to adult-centred care? Best Pract Res Clin Rheumatol 2006;20:387-97.

5. Agarwal S, Raymond JK, Isom S, Lawrence JM, Klingensmith G, Pihoker C, et al. Transfer from paediatric to adult care for young 
adults with Type 2 diabetes: the SEARCH for Diabetes in Youth Study. Diabet Med 2018;35:504-12.

6. McPherson M, Thaniel L, Minniti CP. Transition of patients with sickle cell disease from pediatric to adult care: assessing patient readiness. Pediatr Blood Cancer 2009;52:838-41.

7. Maddux MH, Ricks S, Bass J. Patient and caregiver perspectives of transition and transfer. Clin Pediatr 2017;56:278-83.

8. Howland S, Fisher K. Looking through the patient lens improving best practice for young people with juvenile idiopathic arthritis transitioning into adult care. SpringerPlus 2015;4:11.

9. Tattersall RS, McMahon AM. The MAGICC and practical approach to rheumatology transition. Br J Hosp Med 2012; 73:552-7.

10. Lugasi T, Achille M, Stevenson M. Patients' perspective on factors that facilitate transition from child-centred to adult-centred health care: a theory integrated metasummary of quantitative and qualitative studies. J Adolesc Health 2011;48:429-40.

11. American Academy of Pediatrics, American Academy of Family Physicians, American College of Physicians-American Society of
Internal Medicine. A consensus statement on health care transitions for young adults with special health care needs. Pediatrics 2002;110 Supplement 3:1304-6.

12. Chira P, Ronis T, Ardoin S, White P. Transitioning youth with rheumatic conditions: perspectives of pediatric rheumatology providers in the United States and Canada. J Rheumatol 2014; $41: 768-79$.

13. Matsui T, Matsumoto T, Hirano F, Tokunaga F, Okamoto K, Tohma $S$, et al. Survey of the awareness of adult rheumatologists regarding transitional care for patients with juvenile idiopathic arthritis in Japan. Mod Rheumatol 2018;28:981-5.

14. Foster HE, Minden K, Clemente D, Leon L, McDonagh JE, Kamphuis $S$, et al. EULAR/PReS standards and recommendations for the transitional care of young people with juvenile-onset rheumatic diseases. Ann Rheum Dis 2017;76:639-46.

15. Shaw KL, Southwood TR, McDonagh JE, \& The British Paediatric Rheumatology Group. User perspectives of transitional care for adolescents with juvenile idiopathic arthritis. Rheumatology 2004;43(6):770-8. 